\title{
Enhancement in coercivity of Pulsed Laser Deposition-fabricated Fe-Pt thick film magnets by reducing droplets
}

\author{
M. Nakano, D. Urakawa, ${ }^{\text {a) }}$ T. Yanai, and H. Fukunaga \\ Faculty of Engineering, Nagasaki University, 1-14 Bunkyo-machi, Nagasaki, 852-8521, Japan
}

(Presented 1 November 2011; received 28 September 2011; accepted 15 December 2011; published online 13 March 2012)

\begin{abstract}
A high-speed Pulsed Laser Deposition method with the deposition rate of several ten microns per hour followed by a post-annealing process enabled us to obtain isotropic Fe-Pt thick film magnets with the $\mathrm{L} 1_{0}$ order phase. In the study, we investigated the relationship between droplets and coercivity of the post-annealing films. It was found that the reduction in the numbers of droplets enabled us to increase the coercivity although the average deposition rate decreased from 40 to 15 $\mu \mathrm{m}$ per hour. In the samples with Ra less than $0.5 \mu \mathrm{m}$, the coercivity exceeded $450 \mathrm{kA} / \mathrm{m}$ at the annealing temperature of $673 \mathrm{~K}$. A SEM-EDX analysis of droplets revealed that they tended to have iron-rich or platinum-rich compositions compared with the average one of $\mathrm{Fe}_{50} \mathrm{Pt}_{50}$. C 2012 American Institute of Physics. [doi:10.1063/1.3679566]
\end{abstract}

\section{INTRODUCTION}

Although the high price of platinum is disadvantage in Fe-Pt magnet as an industrial material, the magnet thicker than several microns has been used by several researches who have focused on the outstanding biocompatibility. ${ }^{1,2}$ Watanabe et $_{\mathrm{al}}{ }^{3}$ prepared rapidly-quenched isotropic $\mathrm{Fe}-\mathrm{Pt}$ ribbons with the $(B H)_{\max }$ of approximately $160 \mathrm{~kJ} / \mathrm{m}^{3}$, and Makino et $\mathrm{al}^{4}$ added $\mathrm{Zr}$ and B elements to enhance the coercivity of melt-spun isotropic Fe-Pt alloys up to approximately $650 \mathrm{kA} / \mathrm{m}$. Givord et al. ${ }^{5}$ reported Fe-Pt multilayered foils with the coercivity higher than $800 \mathrm{kA} / \mathrm{m}$ by using a cold-deformation. In addition, sputtering-made isotropic $\mathrm{Fe}$ Pt film magnets thicker than several microns were reported by Aoyama ${ }^{6}$ and Liu, ${ }^{7}$ respectively.

In the paper, isotropic Fe-Pt thick films prepared by using a high-speed Pulsed Laser Deposition (PLD) method with the deposition rate of several-ten-microns per hour. ${ }^{8}$ It is generally said that micrometer-sized droplets are ejected as a whole from a target during a PLD deposition process. In this study, two types of films with different numbers of droplets were fabricated by taking advantage of controlling the focus distance from an optical lens to a target together with the distance between a target and a substrate. This contribution reports that the reduction in the numbers of droplets is effective to increase the coercivity value for the PLD-made isotropic $\mathrm{Fe}-\mathrm{Pt}$ thick film magnets.

\section{EXPERIMENTAL PROCEDURE}

A Fe-Pt target was ablated with a Nd-YAG pulse laser (wave length: $355 \mathrm{~nm}$ ) at the repetition rate of $30 \mathrm{~Hz}$ in a vacuum atmosphere. A laser power was measured with a power meter in front of the entrance lens of a chamber. Before the ablation, the chamber was evacuated down to approximately $4 \times 10^{-7}$ Torr with a rotary pump together

\footnotetext{
${ }^{\text {a) }}$ Author to whom correspondence should be addressed. Electronic mail: bb52111207@cc.nagasaki-u.ac.jp.
}

with a molecular turbo pump. The distance between a target and a Ta substrate (T-S distance) varied from 10 to $20 \mathrm{~mm}$, and the area of all the obtained films were $5 \times 5 \mathrm{~mm}^{2}$. The numbers of droplets in each film were controlled by changing the T-S distance and a DF (Defocus) rate ${ }^{9}$ which was the focus distance between an optical lens and a target. The T-S distance, DF rate and the compositions of targets enabled us to vary the ratio of $\mathrm{Fe}$ to Pt elements in each film. Average deposition rate exceeded $10 \mu \mathrm{m} / \mathrm{h}$, which was almost as twice as that of a sputtering method. ${ }^{6}$

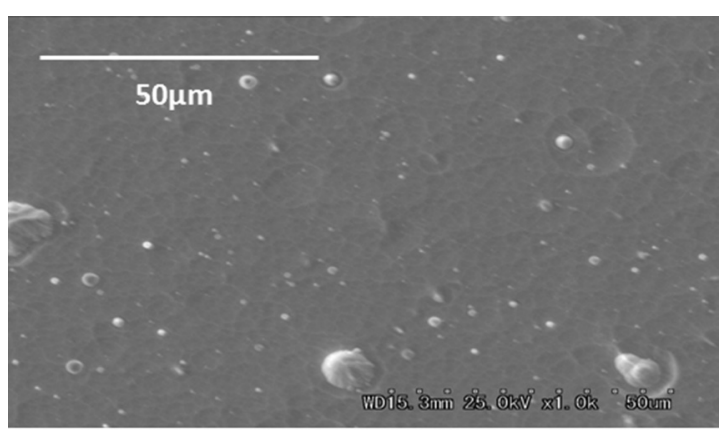

(a). $\mathrm{Ra}=0.44 \mu \mathrm{m}$

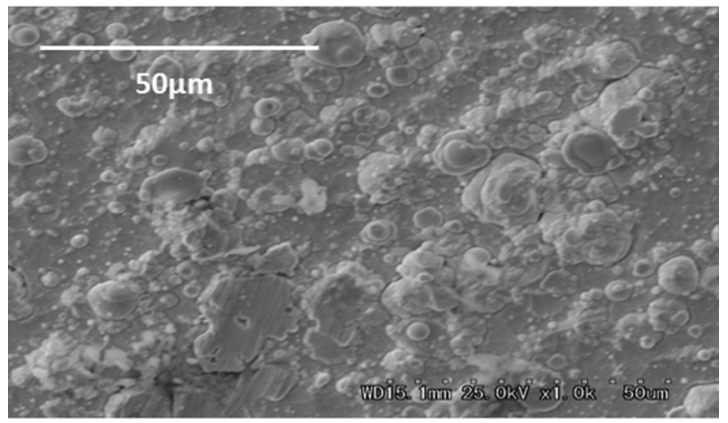

(b). $R a=1.1 \mu \mathrm{m}$

FIG. 1. Surface observations of two samples with the Ra of 0.44 and $1.1 \mu \mathrm{m}$, respectively. A lot of droplets could be observed in the film with the Ra of $1.10 \mu \mathrm{m}$. 


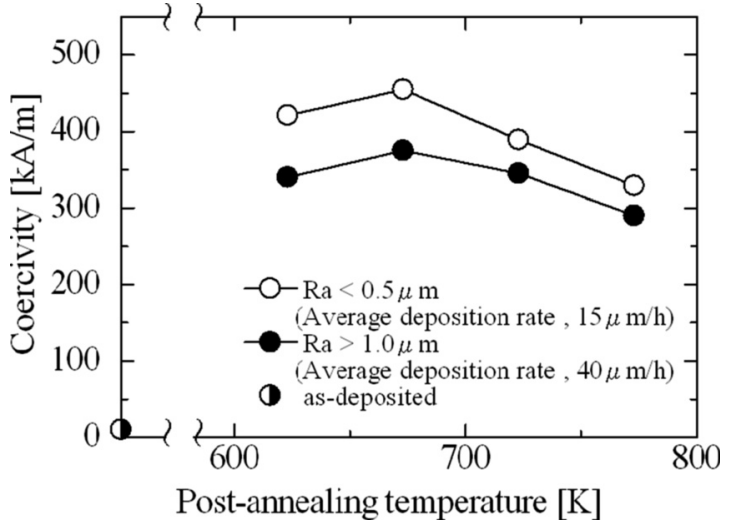

FIG. 2. Coercivity of samples with the Ra less than $0.5 \mu \mathrm{m}$ and larger than $1.0 \mu \mathrm{m}$, respectively, as a function of post-annealing temperature. At the temperature of $673 \mathrm{~K}$, the value became higher than $400 \mathrm{kA} / \mathrm{m}$ in a sample with Ra less than $0.5 \mu \mathrm{m}$.

All the as-deposited film did not have hard magnetic properties because of the existence of the disorder phase, therefore the post-annealing was carried out to obtain the $\mathrm{L} 1_{0}$ order phase. In the annealing process, the rate of temperature rise and holding time were $50 \mathrm{~K} / \mathrm{min}$ and $10 \mathrm{~min}$, respectively, under the range of temperature from 623 to 773 $\mathrm{K}$. After an annealed-sample was magnetized up to $9 \mathrm{~T}$ with a pulse magnetizer, $\mathrm{M}-\mathrm{H}$ loops were measured with a vibrating sample magnetometer (VSM) which could apply a magnetic field up to approximately $1800 \mathrm{kA} / \mathrm{m}$ reversibly. In the experiment, in-plane magnetic properties were only shown because all the films had isotropic magnetic properties. An average thickness was measured with a micrometer. In addi-

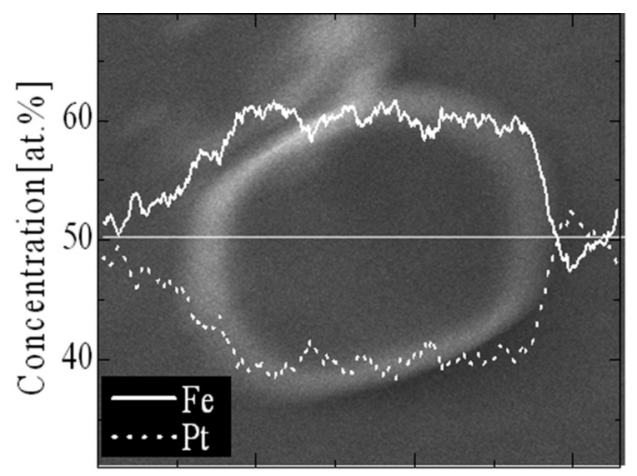

(a). Fe-rich composition

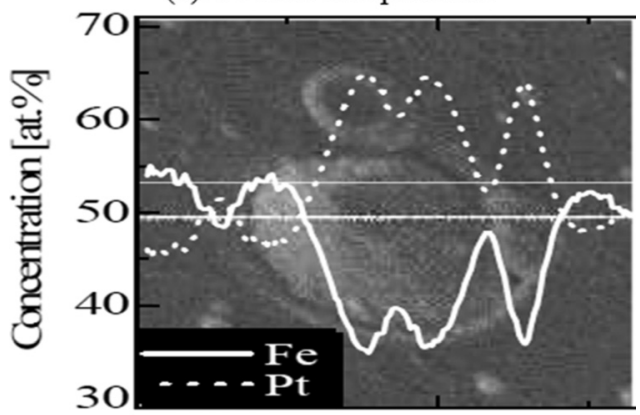

(b). Pt-rich composition

FIG. 3. Compositions of droplets for a $\mathrm{Fe}_{50} \mathrm{Pt}_{50}$ film were evaluated by using a line analysis in SEM-EDX. Droplets with Fe-rich or Pt-rich composition compared to $\mathrm{Fe}_{50} \mathrm{Pt}_{50}$ could be observed.

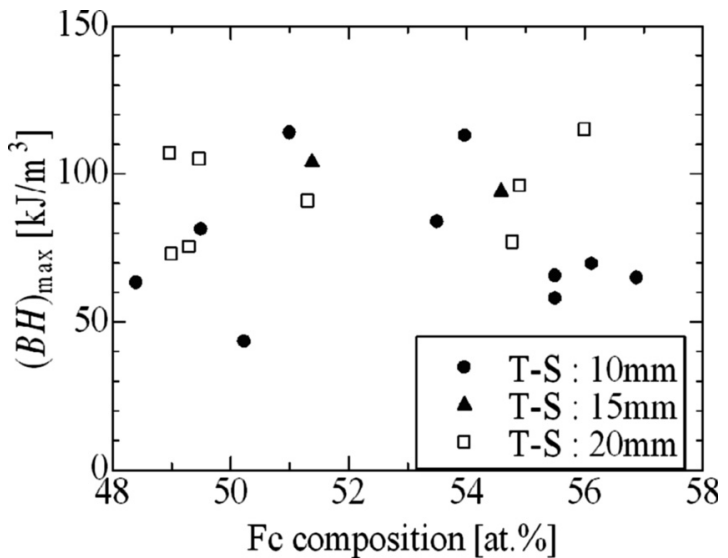

FIG. 4. $(B H)_{\max }$ values as a function of Fe compositions in each sample. The samples were prepared by using $3 \mathrm{~T}-\mathrm{S}$ distances of 10,15 , and $20 \mathrm{~mm}$, respectively.

tion, estimation in the difference of the numbers of droplets was carried out by measuring the centerline average roughness, Ra.

\section{RESULTS AND DISCUSSION}

In order to examine the relationship between the numbers of droplets and magnetic properties, two types of films with the different Ra values were prepared by controlling the $\mathrm{DF}$ rate and T-S distance. Figure 1 shows the surface views of samples with two Ra values of 0.44 and $1.10 \mu \mathrm{m}$, respectively. A lot of droplets could be observed in the film with the Ra of $1.10 \mu \mathrm{m}$. (see Fig. 1 (b)) Coercivity values of samples with two kinds of $\mathrm{Ra}$ as a function of post annealing temperatures were displayed in Fig. 2. Here, the compositions and thicknesses of all films were kept at approximately $\mathrm{Fe}_{50} \mathrm{Pt}_{50}$ and thicker than $10 \mu \mathrm{m}$, respectively. At each temperature, the coercivity values of samples with Ra less than 0.5 were higher than those of Ra larger than $1.0 \mu \mathrm{m}$. In particular, reduction in the numbers of droplets enabled us to obtain samples with the coercivity higher than $400 \mathrm{kA} / \mathrm{m}$ at relatively low temperature of $673 \mathrm{~K}$. An evaluation on compositions of droplets for a $\mathrm{Fe}_{50} \mathrm{Pt}_{50}$ film was carried out by using a line analysis as shown in Fig. 3. In Fig. 3(a), a

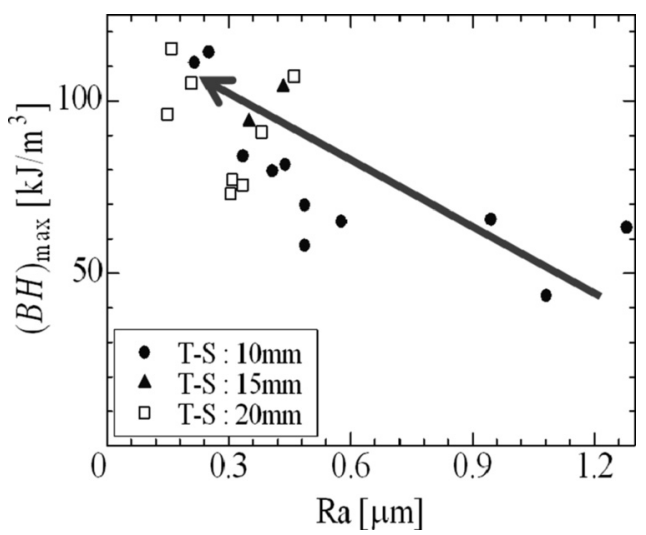

FIG. 5. $(B H)_{\max }$ values as a function of Ra in each sample. The samples were prepared by using $3 \mathrm{~T}-\mathrm{S}$ distances of 10,15 , and $20 \mathrm{~mm}$, respectively. As the value of Ra decreased, $(\mathrm{BH})_{\max }$ increased. 


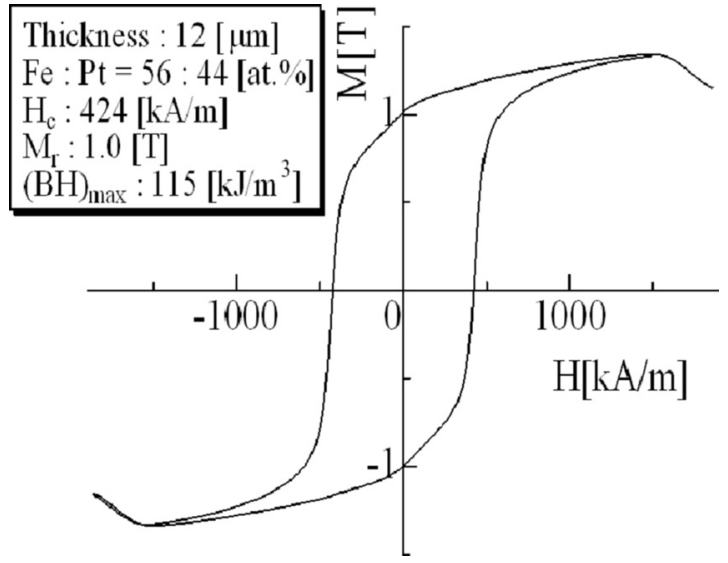

FIG. 6. M-H loop of a Fe-Pt thick film magnet with the Ra of approximately $0.16 \mu \mathrm{m}$. The values of coercivity, remanence and $(B H)_{\max }$ were $424 \mathrm{kA} / \mathrm{m}$, $1.0 \mathrm{~T}$, and $115 \mathrm{~kJ} / \mathrm{m}^{3}$, respectively.

droplet with Fe-rich composition compared to $\mathrm{Fe}_{50} \mathrm{Pt}_{50}$ could be observed. In other droplets, Pt-rich compositions were also observed as shown in Fig. 3(b). The deterioration of magnetic properties due to the increase in the numbers of droplets is considered to be the reduction in the magnetic anisotropy of each film.

Figures 4 and 5 show $(B H)_{\max }$ as a function of Fe compositions and Ra of each sample prepared by $3 \mathrm{~T}-\mathrm{S}$ distances of 10,15 and $20 \mathrm{~mm}$, respectively. In the range of Fe compositions from 48 to 58 at $\%$, the $(B H)_{\max }$ values were independent on the composition. On the other hand, the values of $(B H)_{\max }$ decreased with the reduction in Ra. These results suggest that the magnetic properties of Fe-Pt thick film magnets strongly depend on the numbers of droplets. Resultantly, a Fe-Pt thick film magnet with the Ra of approximately 0.16 $\mu \mathrm{m}$ had the values of coercivity, remanence and $(\mathrm{BH})_{\max }$ of $424 \mathrm{kA} / \mathrm{m}, 1.0 \mathrm{~T}$, and $115 \mathrm{~kJ} / \mathrm{m}^{3}$, respectively, which were comparable to ones of sputtering-made Fe-Pt thick film magnets $^{6,7}$ (see Fig. 6).

\section{CONCLUSION}

In this study, the relationship between the droplets and magnetic properties was investigated in PLD-fabricated FePt thick film magnets. It was found that deterioration of coercivity due to the increase in the numbers of droplets occurs, which is considered to be attributed to the existence of FeRich or Pt-rich droplets compared with the $\mathrm{Fe}_{50} \mathrm{Pt}_{50}$ film composition. By taking advantage of the reduction in the numbers of droplets, a Fe-Pt thick film magnet with the $(B H)_{\max }$ of $115 \mathrm{~kJ} / \mathrm{m}^{3}$ could be obtained under the high deposition rate of $12 \mu \mathrm{m} / \mathrm{h}$.

${ }^{1}$ T. Nakayama, M. Watanabe, M. Homma, T. Kanno, K. Kimura, and O. Okuno, J. Magn. Soc. Jpn. 21, 377 (1997).

${ }^{2}$ A. Yamazaki, M. Sendoh, K. Ishiyama, K. I. Arai, R. Kato, M. Nakano, and H. Fukunaga, J. Magn. Magn. Mater. 272, 1741 (2004).

${ }^{3}$ K. Watanabe and H. Masumoto, Trans. Jpn. Inst. Met. 20, 627 (1983).

${ }^{4}$ A. Makino and T. Bitoh, J. Appl. Phys. 7498, 95 (2004).

${ }^{5}$ N. H. Hai, N. M. Dempsey, and D. Givord, J. Magn. Magn. Mater. 262, 353 (2003).

${ }^{6}$ H. Aoyama and Y. Honkura, J. Magn. Soc. Jpn. 20, 237 (1996).

${ }^{7}$ W. F. Liu, S. Suzuki, D. S. Li, and K. Machida, J. Magn. Magn. Mater. 302, 201 (2006).

${ }^{8}$ M. Nakano, W. Oniki, T. Yanai, and H. Fukunaga, J. Appl. Phys. 109, 07 A723 (2011).

${ }^{9}$ H. Fukunaga, T. Kamikawatoko, M. Nakano, T. Yanai, and F. Yamashita, J. Appl. Phys. 109, 07A758 (2011). 